\title{
A GLOBAL EXISTENCE THEOREM FOR THE FOUR BODY PROBLEM ${ }^{1}$
}

\author{
BY DONALD G. SAARI \\ Communicated by Robert Seeley, May 7, 1976
}

Consider the four body problem of Newtonian mechanics where physical space is either two or three dimensional and where the center of mass of the system is fixed at the origin of the inertial coordinate system. Phase space is 12 dimensional for the planar problem and 18 dimensional for the nonplanar problem. One of the basic questions in the study of the 4 body problem is to determine the size and nature of the set of initial conditions for which the solutions terminate in finite time.

THEOREM. Let $B$ be the set of initial conditions such that an analytic solution of the 4 body problem exists on the domain $(-\infty, \infty)$. The complement of $B$ is a set of Lebesgue measure zero and first Baire category.

A similar result is known to hold for the 2 and 3 body problems. This leads to the following statement.

COROllary. For almost all initial conditions of the noncollinear 2, 3, or 4 body problems, an analytic solution exists for all values of time.

The analysis for the 2 and 3 body problem differs sharply from that of the $n$ body problem for $n \geqslant 4$. It was shown by Painlevé [4] that the only possible singularities for the 2 and 3 body problems are collisions. It was conjectured by J. Littlewood [1], [2] and shown by Saari [5], [6], [7] that for the noncollinear $n$ body problem the set of initial conditions leading to collisions forms a set of Lebesgue measure zero and first Baire category. Because of these results, the announced theorem follows if the set of initial conditions leading to noncollision singularities can be shown to be small in the above sense. This is what is done. (The existence of noncollision singularities remains an open question. However a recent related result by Mather and McGehee [3] suggests the answer may be in the affirmative. They show that such motion does exist in the collinear four body problem if binary collisions are admitted.)

This analysis is aided by a characterization of noncollision singularities in the $n$ body problem given in $[11],[10],[8]$. In particular, for the four body

AMS (MOS) subject classifications (1970). Primary 70F10, 70F35; Secondary 34F05, 58 F05.

1 This research was partially supported by an NSF grant.

Copyright $\odot 1976$, American Mathematical Society 
problem, it follows from what was shown in [8] that the particles oscillate wildly, the system becomes unbounded in finite time, but the particles must approach a fixed line in physical space. (It has been called to my attention that a similar statement has been given by R. Shelton in his Rice University dissertation.) The basic idea of the proof, which will appear elsewhere, is to use these facts to determine how fast the particles must approach this line.

Since the system is measure preserving, these estimates provide a bound on the measure of the initial conditions leading to this wildly oscillating system. It turns out that this bound can be made arbitrarily small, showing that the set of initial conditions leading to this type of behavior forms a set of Lebesgue measure zero. A standard argument using this measure zero result and continuity of solutions with respect to initial conditons obtains the Baire category statement.

Based on the behavior of this system, it seems very likely that a similar global existence theorem holds for the noncollinear $n$ body problem for $n \geqslant 5$.

As part of the analysis, it turns out that if noncollision singularities exist, then certain relationships between the masses must exist. A special case of the contrapositive of this statement follows.

Corollary. Consider the four body problem where $m_{1}=m_{2}=m_{3}=$ $m_{4}=m>0$. Then any singularity is due to collisions.

In this case, the earlier statement asserting the improbability of collisions applies.

\section{REFERENCES}

1. J. E. Littlewood, Some problems in real and complex analysis, Heath, Lexington, Mass., 1968. MR 39 \#5777.

2. A mathematician's miscellany, Methuen, London, 1953.

3. J. Mather and R. McGehee, Solutions of the collinear four body problem which become unbounded in finite time, Dynamical Systems, Battelle Rencontres (Seattle, 1974), Springer-Verlag, Berlin, 1975.

4. H. Painlevé, Leçons sur la théorie analytique des équations différentielles, Hermann, Paris, 1897.

5. D. G. Saari, Improbability of collisions in Newtonian gravitational systems, Trans. Amer. Math. Soc. 162 (1971), 267-271; erratum, ibid. 168 (1972), 521. MR 45 \#4714.

6. - Improbability of collisions in Newtonian gravitational systems. II, Trans. Amer. Math. Soc. 181 (1973), 351-368. MR 47 \#9919.

7. - Collisions are of first category, Proc. Amer. Math. Soc. 47 (1975), $442-$ 445. MR 51 \#2395.

8. Singularities and collisions of Newtonian gravitational systems, Arch. Rational Mech. Anal. 49 (1972/73), 311-320. MR 49 \#4354.

9. - Singularities of Newtonian gravitational systems, Dynamical Systems (Proc. Sympos., Univ. of Bahia, Salvador, 1971), M. Peixoto, Editor), Academic Press, New York, 1973, pp. 479-487. MR $49 \# 10210$.

10. H. J. Sperling, On the real singularities of the $N$-body problem, J. Reine Angew Math. 245 (1970), 15-50. MR 44 \#7810.

11. H. von Zeipel, Sur les singularités du problème des $n$ corps, Ark. Mat. Astr. Fys. 4 (1908), no. 32 . 